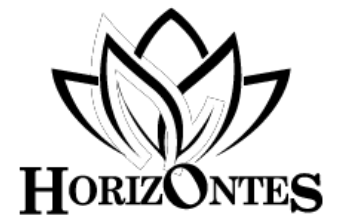

Horizontes. Revista de Investigación en Ciencias de la Educación Https://doi.org/10.33996/revistahorizontes.v2i7.53 julio - septiembre, 2018 Volumen 2 / No. 7 pp. $154-170$

www.revistahorizontes.org

\title{
Calidad académica de los docentes universitarios
}

\author{
Academic quality of the college professors
}

\author{
Wendy Montoya \\ carolinew_82@hotmail.com
}

Universidad Politécnica Territorial del Norte del Táchira Manuela Sáenz

\section{RESUMEN}

La educación universitaria es la base para el desarrollo social, científico, tecnológico y humanístico, por lo tanto, se debe generar una gestión eficaz y eficiente en el desarrollo académico que ejercen los profesores. En tal sentido, la presente investigación tuvo como objetivo general describir la calidad académica de los docentes de la Universidad Politécnica Territorial del Norte del Táchira Manuela Sáenz (UPTNT Manuela Sáenz). Asimismo, se abordaron las perspectivas teóricas a través de otros estudios, leyes y autores que dan sustento a la investigación. Por tal motivo, la investigación parte del proceso sintagmático, bajo el tipo de investigación descriptiva. El diseño de la investigación fue transeccional contemporáneo de campo, univariable, con un abordaje cosmológico, étic, exógeno. Se trabajó con una población conformada por 70 docentes de la insti-tución universitaria. La técnica de recolec-ción de los datos estuvo conformada por un instrumento tipo escala con 130 ítems, la validez se realizó a través del juicio de expertos y la confiabilidad por medio del programa estadístico SPSS, dando como resultado ,820. Se concluyó que los docentes de la universidad en estudio presentan una calidad académica regular, es decir, hay poca pertinencia, exhaus-tividad, innovación, efectividad e idoneidad en el quehacer académico.

Palabras clave: calidad académica; educación; educación universitaria

\begin{abstract}
College education is the base of the social, scientific, technological and humane development, as such, an effective and competent management must be put in place in the academic development exercised by the professors. On that regard, the following investigation has as purpose to describe the academic quality of the professors of the Northern Tachira Territories Polytechnic University Manuela Sáenz (UPTNT Manuela Sáenz). Likewise, the theoretical perspectives were addressed through other studies, laws and authors that support the research. For this reason, the research starts from the syntagmatic process, under the type of descriptive research. The design of the research was transeccional contemporary field, univariable, with a cosmological, etic, exogenous approach. The population was made up of 70 teachers from the university. The data collection technique consisted of a scale-type instrument with 130 items, the validity was made through expert judgment and reliability through the statistical program SPSS, with a result of 820 . It was concluded that the teachers of the university under study present a regular academic quality, that is to say, there is little pertinence, completeness, innovation, effectiveness and suitability in the academic task.
\end{abstract}

Key words: academic quality; education; university education 


\section{INTRODUCCIÓN}

El hombre es un ser social por naturaleza, es ahí, donde radica su necesidad de aprender y conocer constantemente, en tal sentido, su deseo de superación personal y profesional lo motivan a continuar en la búsqueda de aquello que lo inquieta y lo convierte en un ser con talentos y virtudes que desarrolla día tras día. Pero no es sino a través de la formación escolar que el hombre adquiere las herramientas que desarrollan sus capacidades intelectuales, culturales, humanísticas, espirituales, sociales, políticas, científicas, entre muchas otras, de acuerdo con su vocación, interés, necesidad o pasión.

De este modo, la educación como dadora de conocimiento académico, científico, tecnológico y humanístico, necesita que los directores, rectores y profesores se manifiesten en ir hacia la excelencia de la calidad educativa, en este caso citando a Kant (2008), dice:

La educación es un arte cuya ejecución tiene que ser perfeccionada por muchas generaciones. Cada generación, dotada de los conocimientos de la precedente, puede cada vez más poner en efecto una educación que desarrolle proporcional y adecuadamente todas las aptitudes naturales del hombre, y lleve así a todo el género humano a su destino. (p. 35).

Desde esta perspectiva, los docentes tienen la labor de mejorar el proceso educativo a través de nuevos conocimientos que permitan contribuir en distintas áreas del saber, de esta manera se nutre la academia y se puede ejercer un criterio de calidad educativa.

Por tanto, la educación, específicamente la universitaria desempeña un papel importante en la formación de las personas porque reviste el desarrollo de todas las disciplinas para la construcción de nuevos aprendizajes y conocimientos. De la Fuente (2008, p. 22) expresa que "el desafío de la universidad es construir una escuela para el futuro y el futuro de la universidad".

Dentro de este marco, la educación universitaria proporciona oportunidades al individuo, es por esta razón que, la responsabilidad del trabajo académico es una visión compartida de todos los que la conforman, a fin de estar a la par de los constantes retos científico y tecnológico que se presentan.

En la actualidad, las universidades tienen la tarea de hacer frente a diversas vicisitudes que afectan a la sociedad y que trascienden las generaciones, desde esta inquietud, las instituciones universitarias están llamadas a contribuir con las mejoras académicas, quiere decir que, desde los aportes de los profesores en cuanto a conocimientos científicos, innovación y preparación continua del mismo docente, la institución universitaria se fortalece académicamente.

En este sentido, la universidad en su carácter de promover y fortalecer la academia debe afianzar el trabajo en sus áreas primordiales, como son: la docencia, la investigación y la extensión, por tanto, no debe estar aislada una de la otra porque juntas solidifican la existencia dentro del espacio académico y se apoyan en la construcción de nuevos conocimientos que permite poner en marcha la creatividad, motivación de los profesores y por ende también de los estudiantes.

En razón de lo planteado, Llorens (2013, p. 15) manifiesta, que la institución debe ser "una universidad eficiente y de calidad que incentive la formación de sus profesores y de su personal de apoyo, que se comprometa... y que, en definitiva, impulse y estimule una educación más activa". Es decir, debe centrarse en la libertad de pensamientos, de ideas, para producir y aportar a una sociedad sumergida en un contexto de innovación cons- 
tante, donde profesores como estudiantes se sientan familiarizados y trabajen para lograr el reconocimiento de la excelencia académica.

A tal efecto, llegar a la calidad académica es un trabajo en equipo que se realiza con todos los actores del proceso académico como: las autoridades universitarias, profesores, estudiantes y todo aquel que esté involucrado directa e indirectamente con la academia.

En Venezuela, el proceso educativo universitario comienza en el siglo XVII con la creación de universidades que hoy día son ícono en la educación del país.

En Venezuela, la llegada del siglo XXI marca una serie de transformaciones políticas y trae consigo una perspectiva nueva en la educación, haciendo énfasis en la universitaria que tiene como objetivo la universalización de la educación y un nuevo modelo educativo.

Es así como en el país hoy día funcionan un número de universidades agrupadas en: nacionales autónomas, nacionales experimentales, privadas, institutos y colegios privados; además de las creadas por el gobierno actual como: las universidades bolivarianas que se ubican en aproximadamente veinte estados del país; la Misión Sucre conformada en todos los estados y municipios del país y los institutos universitarios y colegios universitarios que en su mayoría pasaron a ser universidades politécnicas territoriales.

Sin embargo, si bien es cierto que la proliferación de instituciones universitarias favorece la inclusión de las personas al sistema educativo sin ningún rechazo y discriminación, también lo es que represente para la educación un desafío en cuanto a cantidad por calidad o calidad por cantidad, dilema que conduce a las universidades a seguir en la lucha de garantizar una educación con principios, de calidad y de producción científica.
En este contexto, Águila (2005, p. 4) afirma que,

...el concepto de la calidad... tiene la dificultad de que pueda no ser suficiente para garantizar la calidad de la universidad si los propósitos son limitados, pobres y regionales. Pero posee la ventaja de que un país o una institución pueden trazarse y luchar por sus propias metas sobre la base de sus aspiraciones. (p. 4).

Por lo tanto, para que predomine la calidad académica en las instituciones universitarias, deben existir factores que trabajen de forma cónsona y no de manera aislada y que estén enfocados en la actividad de todos los que participan dentro del recinto universitario porque cada uno tiene sus funciones, además del compromiso de generar aportes que alcancen el logro de la calidad académica, aun cuando trabajar para llegar a ella, sea complejo en el cumplimiento de su totalidad.

De igual manera, se hace necesario resaltar que la calidad en la educación actúa con componentes que la llevan a alcanzar resultados satisfactorios, (Pérez, 2002, c.p Montes, 2013) manifiesta tres indicadores que acompañan al concepto de calidad educativa: eficacia, relevancia y, procesos y medios; el primero de los indicadores citados corresponde a los planes y programas curriculares; la relevancia, se refiere a la correspondencia de los contenidos con lo que el individuo necesita para desarrollarse como persona, y por último, los procesos y medios, basados en la preparación del cuerpo de docentes para enseñar.

Sobre la base de la observación, Venezuela está haciendo frente a una crisis socioeconómica que, a su vez, afecta con predominio a las universidades, esto hace que el proceso de enseñanza aprendizaje no se genere 
como es debido, por la precariedad de recursos económicos y de implementos necesarios en las diferentes áreas del saber. Además, se presenta también el desinterés o desmotivación por parte de los entes dadores de conocimiento, como lo son los docentes, a tal fin que no puedan lograr sus propios objetivos y no se hable de calidad en la educación y por ende, el trabajo no se realiza con eficiencia y decae la calidad académica.

A tal efecto, los mismos docentes y estudiantes se sienten preocupados en cuanto a la calidad académica en el ámbito universitario, ya que en las universidades cada día la deserción estudiantil y profesoral es predominante, lo cual deteriora la calidad de una educación productiva que genere talentos de acuerdo con las necesidades no solo nacionales, sino globales.

En el contexto de estudio, la Universidad Politécnica Territorial del Norte del Táchira Manuela Sáenz, es una institución concebida como Universidad Politécnica Territorial el 16 de julio de 2010 consagrada en el decreto presidencial $\mathrm{N}^{\circ} 7.570$, bajo la gaceta oficial extraordinaria $\mathrm{N}^{\circ} 5987$.

Sin embargo, dicha universidad no escapa de los problemas que afectan a la sociedad y que recaen paulatinamente en la educación. A través de la misma base de la observación, se determinan varias dificultades que coartan el incremento del potencial académico y se convierten en obstáculo para el desarrollo intelectual que debe persistir en la academia.

Actualmente, en la UPTNT Manuela Sáenz, se observa apatía por parte de los docentes hacia el trabajo académico y la poca voluntad para un trabajo en equipo que permita el crecimiento de la universidad, lo cual ha traído como consecuencia: deserción estudiantil, poca producción investigativa, poca participación en las actividades de formación permanente que imparte la universidad, es decir, hay poco desarrollo de una perspectiva crítica que conduzca hacia la transformación universitaria y que permita el logro de objetivos, lo cual provoca una monotonía pedagógica.

Asimismo, los profesores no asisten a eventos académicos fuera de la institución, además no existe la articulación de la UPTNT Manuela Sáenz con otras instituciones universitarias para la integración de conocimientos en pro de la consolidación y el buen hacer académico.

En tal sentido, la institución enfrenta un declive en cuanto a la calidad académica y en función de este problema, surge la siguiente interrogante, ¿Cómo es la calidad académica de los docentes de la Universidad Politécnica Territorial del Norte del Táchira Manuela Sáenz?

Ante dicha inquietud se plantea como objetivo general en esta investigación: Describir la calidad académica de los docentes de la Universidad Politécnica Territorial del Norte del Táchira Manuela Sáenz.

Los inicios de la educación universitaria en Venezuela se remontan al siglo XVIII con la creación de la Universidad Santiago de León, conocida hoy día como la Universidad Central de Venezuela. Al transcurrir el tiempo se crearon otras universidades en algunos estados del país, como Mérida con la Universidad de Los Andes, Maracaibo con la Universidad del Zulia.

Para los años de 1960, exponen Morales, Medina y Álvarez (2003), se crean las universidades experimentales. En tal sentido que comienza a expandirse la educación universitaria por el territorio nacional, abriendo las posibilidades de estudio a las personas.

Por consiguiente, a finales del siglo XX y principios del siglo XXI Venezuela comenzó un periodo de transición que condujo a cambios políticos, económicos, sociales, culturales y educativos; es así como la educación da un vuelco y se empiezan a generar otras formas 
de ver el proceso de educación, en todos los niveles, desde la educación inicial hasta la universitaria, dando aún más oportunidades para el ingreso a la educación.

En tal sentido, la Constitución de la República Bolivariana de Venezuela (1999) en su capítulo VI de los derechos culturales y educativos, en el artículo 103 expresa:

Toda persona tiene derecho a una educación integral, de calidad, permanente, en igualdad de condiciones y oportunidades, sin más limitaciones que las derivadas de sus aptitudes, vocación y aspiraciones. La educación es obligatoria en todos sus niveles... La impartida en la institución del estado es gratuita hasta el pregrado universitario (p. 17).

Esta nueva visión sobre el proceso educativo conlleva a la inclusión de un gran número las personas al sistema educativo, sin ningún rechazo y discriminación debido a la cantidad de universidades que comienzan a crearse a lo largo del territorio nacional.

Es así como, el gobierno crea la Misión Alma Mater con el objetivo de arraigar la educación superior en todo el territorio nacional, en estrecho vínculo con las comunidades, en tal sentido, dicha misión dio lugar a la creación de universidades direccionadas directamente por el gobierno como: la Universidad Bolivariana de Venezuela y las Universidades Politécnicas Territoriales, las cuales se encuentran ubicadas en diferentes espacios del territorio nacional y donde deben dar respuestas científicas a las necesidades que se presenten.

Por consiguiente, en Venezuela se encuentra un número de instituciones de educación universitaria y se pueden clasificar de la manera siguiente: Universidades: autónomas, experimentales, privadas politécnicas. Institutos y colegios universitarios: institutos politécnicos, institutos pedagógicos, institutos universitarios de tecnología, colegios universitarios, institutos universitarios eclesiásticos e institutos militares universitarios.

De esta manera, cada recinto universitario cumple su función académica desde sus propios paradigmas, sus enfoques, normativas y reglamentos que los ponen al servicio de la población, como así lo establece la Ley de Universidades $(1970, p, 1)$ en su artículo 2 "Las universidades son instituciones al servicio de la Nación y a ellas corresponde colaborar en la orientación de la vida del país mediante su contribución doctrinaria en el esclarecimiento de los problemas nacionales", logrados a través de la buena gestión para el crecimiento académico.

Sin embargo, las universidades hoy día se enfrentan a retos fundamentales, Salas (2000, p. 38) los menciona, como: "equidad, calidad y eficiencia. Flexibilidad curricular, pertinencia social e investigación profesional y estudiantil. Inserción, interacción y compromiso social de la universidad con el país. Modernización y descentralización de los procesos académicos-administrativos".

De tal manera que, a pesar de las dificultades presentes en las universidades, estas deben trabajar para solidificar la educación y llevarla a un nivel de excelencia en su calidad académica, pues es lo que determina el éxito institucional.

De acuerdo al diccionario de etimología, la palabra calidad viene del latín qualitas formada con: el interrogativo quae (qué) (interrogación), el sufijo alis (relativo) y el sufijo tat (denota cualidad). Según la Real Academia Española, define calidad como "propiedad o conjunto de propiedades inherente a algo que permiten juzgar su valor".

Asimismo, Crosby (1987) se refiere a la calidad como un catalizador muy importante 
que establece las diferencias entre el éxito y el fracaso, por tanto, todas las cosas se deben hacer bien desde la primera vez y debe formar parte de la vida cotidiana.

El término calidad dentro de la educación, en la actualidad es un tema que se debate constantemente, ya que las instituciones educativas quieren alcanzar ciertos estándares de calidad. La calidad de la educación hace referencia a la gestión externa que realizan los entes encargados para garantizar un adecuado sistema educativo, Vásquez (2013, p. 58) dice "la calidad de la educación conlleva posicionamiento político, social y cultural frente a lo educativo".

Sin embargo, hoy día la educación enfrenta una crisis, por lo tanto, es conveniente preguntar ¿Es viable hablar de calidad de la educación?, pues los problemas políticos, sociales y culturales, afectan a la calidad, repercuten en la labor institucional y no se pone de manifiesto la calidad educativa, las instituciones están en una situación agonizante del que tratan de surgir pese a las circunstancias.

La afirmación anterior, se sustenta con lo que expresa Cano (1998, p. 9), cuando señala: "la calidad de la educación forzosamente deberá referirse a la multiplicidad de elementos del proceso educativo, desde la amplitud de objetivos de la educación a la variedad de elementos intervinientes"; la calidad de la educación no solo hace referencia a los procesos, sino también, al cumplimiento de cada uno de ellos, a la actividad pedagógica, asimismo, al compromiso de los estudiantes, porque para lograr la calidad educativa es necesaria la intervención de todos sus actores.

En el mismo orden de ideas, las autoras Vicuña y Hurtado $(2014$, p. 25) crean un sintagma del término de calidad y estudian la calidad metodológica donde dicen "la calidad metodológica de la investigación se expresa en la medida en que el proceso es idóneo, coherente, exhaustivo, pertinente, original y acorde con las normas de presentación", en tal sentido, desde una perspectiva holística, permite integrar los aspectos nucleares de una investigación que lleva al entendimiento, originalidad y calidad del producto final.

Del mismo modo, la Unesco (2008, p, 15) en la Conferencia Regional de Educación Superior en América Latina y el Caribe de 1996, expresó que la academia "no puede concebirse desligada de la calidad. Pertinencia y calidad, se dijo, están estrechamente ligadas, son conceptos interdependientes, como las dos caras de una misma moneda". Al respecto, Salas (2000) expresa que:
La calidad académica no es una abstracción, sino un referente social e institucional y sus resul- tados tienen que ser analizados, no solo en términos cognos- citivos y conductuales, sino en cuanto a la producción intelec- tual y científica, y cómo dan respuestas a las universi-dades, a las necesidades plantea-das por el encargo social. (p. 137)

De tal manera, la presente investigación plantea la calidad académica desde una explicación sintagmática que a su vez la acompañan aspectos claves y predominantes en la educación activa, productiva e integradora que permite el profesionalismo de los docentes y estudiantes al formar parte de la sociedad del conocimiento. Estos aspectos son sinergias que surgen del estudio cauteloso del evento calidad académica y son: la pertinencia, la exhaustividad, la innovación, la efectividad y la idoneidad.

Cabe señalar que, las sinergias pertinencia, exhaustividad e idoneidad, son aspectos nucleares en el trabajo de las investigadoras citadas, Vicuña y Hurtado (2014). Por lo tanto, en la presente investigación, dichas sinergias 
se trabajan desde la calidad académica y también se integran otras como, innovación y efectividad.

\section{MÉTODO}

La investigación se desarrolla en el estadio descriptivo por cuanto en este caso se pretende describir la calidad académica de los docentes de la UPTNT Manuela Sáenz.

El diseño de la investigación es de campo, transeccional contemporáneo. Para la recolección de los datos se utiliza la técnica de la encuesta con un cuestionario identificado como escala de calidad académica, el cual se aplica a 70 docentes de la institución universitaria. El análisis de los datos se realiza con la estadística de análisis descriptivo: mediciones, frecuencias y porcentajes. Los resultados se interpretan con el baremo de interpretación que se presenta en el cuadro 1.

Cuadro 1. Baremo de interpretación

\begin{tabular}{ll}
\hline Categoría & Interpretación \\
\hline 0 a 19,9 & Muy baja Calidad \\
20 a 39,9 & Baja Calidad \\
40 a 59,9 & Mediana Calidad \\
60 a 79,9 & Alta calidad \\
80 a 100 & Muy alta calidad \\
\hline
\end{tabular}

RESULTADOS

La descripción general del evento calidad académica es determinada a partir del cuadro de puntaje bruto transformado, la mediana y el gráfico de caja donde se muestran los valores mínimos y máximos del evento estudiado.

Los datos presentados en el cuadro 2, muestran una mediana de 41,923 en una escala de 0 a 100 puntos, por lo que dicho pun- taje se ubica, según el baremo de interpretación, en mediana calidad académica. De tal manera que, puede inferirse que existe un déficit en la calidad académica de los profesores que imparten sus unidades curriculares en la casa de estudio, es decir, el trabajo no lo realizan en su totalidad tomando en consideración la pertinencia, la exhaustividad, la innovación, la efectividad y la idoneidad.

Cuadro 2. Mediana de calidad académica

\begin{tabular}{llr}
\hline N & $\begin{array}{l}\text { Válidos } \\
\text { Perdidos }\end{array}$ & $\mathbf{7 0}$ \\
& & $\mathbf{0}$ \\
\hline Mediana & & 41,923 \\
Mínimo & & 29,2 \\
Máximo & & 45,6 \\
Percentiles & 25 & 40,529 \\
& 50 & 41,923 \\
& 75 & 43,510 \\
\hline
\end{tabular}


En el gráfico de caja y bigote (gráfico 1), se presenta un puntaje mínimo de 29,2 y un máximo de 45,6. Los datos se distribuyen asimétricamente, ante lo cual se puede afirmar que el grupo es homogéneo, es decir, presenta características similares.

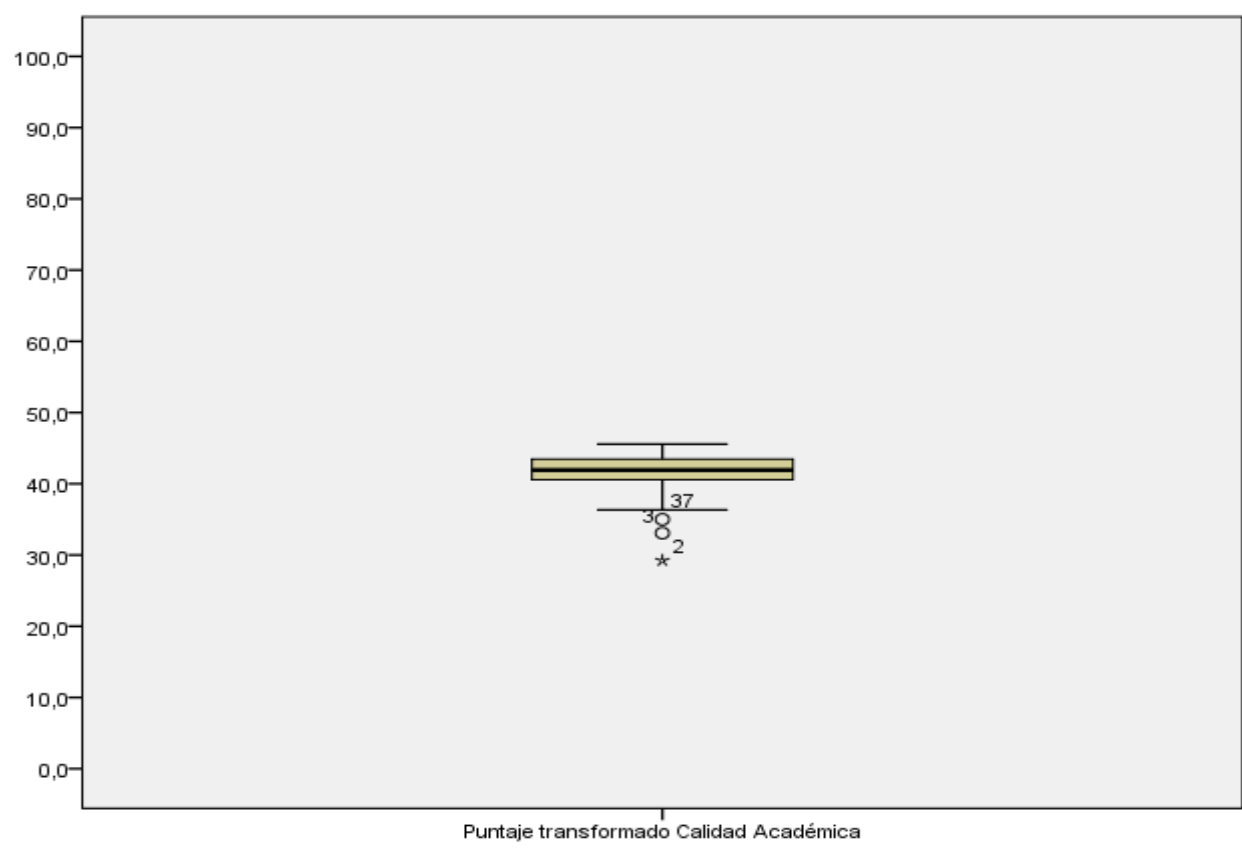

Gráfico 1. Caja y bigote del evento calidad académica.

En el cuadro 3 se presenta la distribución de la población en las categorías de calidad académica donde se observa que el $20 \%$ de los docentes se ubican en una calidad acadé- mica deficiente y el mayor porcentaje recae en la categoría calidad académica regular con el $80 \%$ de los docentes.

Cuadro 3. Categoría de calidad académica

\begin{tabular}{llcccc}
\hline & Frecuencia & Porcentaje & $\begin{array}{c}\text { Porcentaje } \\
\text { válido }\end{array}$ & $\begin{array}{c}\text { Porcentaje } \\
\text { acumulado }\end{array}$ \\
\hline \multirow{3}{*}{ Válidos } & Calidad deficiente & 14 & 20,0 & 20,0 & 20,0 \\
& Calidad regular & 56 & 80,0 & 80,0 & 100,0 \\
& Total & 70 & 100,0 & 100,0 & \\
\hline
\end{tabular}

Análisis de las sinergias del evento calidad académica

En el cuadro 4 se puede apreciar las sinergias que conforman el evento calidad académica (pertinencia, exhaustividad, innovación, efectividad e idoneidad), donde puede observarse que, según el baremo de interpre- tación, todas las sinergias se encuentran en una categoría de mediana calidad académica.

Estos resultados indican con claridad que medianamente los docentes emplean la pertinencia, la exhaustividad, la innovación, la efectividad y la idoneidad en el trabajo académico. 
Cuadro 4. Sinergias del evento calidad académica

\begin{tabular}{lrrrrrr}
\hline & $\begin{array}{l}\text { Puntaje } \\
\text { transformado } \\
\text { pertinencia }\end{array}$ & $\begin{array}{l}\text { Puntaje } \\
\text { transformado } \\
\text { exhaustividad }\end{array}$ & $\begin{array}{l}\text { Puntaje } \\
\text { transformado } \\
\text { innovación }\end{array}$ & $\begin{array}{l}\text { Puntaje } \\
\text { transformado } \\
\text { efectividad }\end{array}$ & $\begin{array}{l}\text { Puntaje } \\
\text { transformado } \\
\text { iddoneidad }\end{array}$ \\
\hline N Validos & & 70 & 70 & 70 & 70 & 70 \\
Perdidos & $\mathrm{P}$ & 0 & 0 & 0 & 0 & 0 \\
Mediana & & 42,500 & 42,5000 & 41,667 & 41,250 & 43,750 \\
Mínimo & & 21,7 & 28,75 & 25,0 & 28,3 & 31,3 \\
Máximo & & 51,7 & 51,25 & 48,3 & 49,2 & 52,5 \\
Percentiles & 25 & 40,000 & 38,7500 & 38,125 & 37,500 & 40,000 \\
& 50 & 42,500 & 42,5000 & 41,667 & 41,250 & 43,750 \\
& 75 & 45,208 & 45,0000 & 44,375 & 43,333 & 46,250 \\
\hline
\end{tabular}

Con respecto a la sinergia pertinencia, según el cuadro 4, la mediana es de 42,5 puntos de un máximo de 100 puntos, esto indica que medianamente existe pertinencia en la correspondencia que el docente hace de los contenidos del programa nacional de formación con las necesidades del contexto.

La distribución de la población en las ca- tegorías de la sinergia pertinencia se presenta en el cuadro 5 y demuestra que el $78,57 \%$ de los docentes presentan una mediana pertinencia en el trabajo académico realizado en la institución universitaria, mientras que un $21,4 \%$ de los docentes muestran una calidad académica deficiente.

Cuadro 5. Categoría de la sinergia pertinencia

\begin{tabular}{llcccc}
\hline & Frecuencia & Porcentaje & $\begin{array}{c}\text { Porcentaje } \\
\text { válido }\end{array}$ & $\begin{array}{c}\text { Porcentaje } \\
\text { acumulado }\end{array}$ \\
\hline \multirow{2}{*}{ Válidos } & Calidad deficiente & 15 & 21,4 & 21,4 & 21,4 \\
& Calidad regular & 55 & 78,6 & 78,6 & 100,0 \\
& Total & 70 & 100,0 & 100,0 & \\
\hline
\end{tabular}

Los aspectos desfavorables de la sinergia pertinencia, correspondieron a que un $69 \%$ los profesores manifestaron no tomar en cuenta las necesidades del entorno donde se encuentra la universidad, en tal sentido, la labor académica está alejada de los acontecimientos que ocurren en el entorno universitario.

Aunado a eso, un $67 \%$ manifestó que rara vez utiliza recursos didácticos para dar solución a las necesidades del contexto donde está la universidad.

También en su mayoría, un $72 \%$ de los profesores respondieron que rara vez promueven la integración de sus compañeros a través de técnicas educativas para la mejora académica dentro de la comunidad universitaria.

Asimismo, en un 68\% los profesores rara vez trabajan con los recursos didácticos que presenta el diseño curricular para las evaluaciones académicas, en tanto, los profesores prefieren trabajar con otros recursos didácticos que no aparecen en el diseño curricular de su programa nacional de formación.

\section{Análisis de la sinergia exhaustividad}

Con referencia a la sinergia exhaustividad, en el cuadro 4 se observa que la misma muestra una mediana de 42,5 puntos de un máximo de 100 , lo que la ubica en una categoría de mediana calidad. Por lo tanto, puede 
afirmarse que los profesores desempeñan un trabajo medianamente exhaustivo, puesto que esta sinergia hace alusión al trabajo del docente de manera profunda y extensa.

Cuadro 6. Categorías de exhaustividad

\begin{tabular}{|c|c|c|c|c|c|}
\hline & & Frecuencia & Porcentaje & $\begin{array}{l}\text { Porcentaje } \\
\text { válido }\end{array}$ & $\begin{array}{l}\text { Porcentaje } \\
\text { acumulado }\end{array}$ \\
\hline \multirow[t]{3}{*}{ Válidos } & $\begin{array}{l}\text { Calidad deficien- } \\
\text { te }\end{array}$ & 21 & 30,0 & 30,0 & 30,0 \\
\hline & Calidad regular & 49 & 70,0 & 70,0 & 100,0 \\
\hline & Total & 70 & 100,0 & 100,0 & \\
\hline
\end{tabular}

De igual manera, en el cuadro 6 se representa la distribución de la categoría exhaustividad, en ella se observa que un $30 \%$ de los profesores mantienen una calidad deficiente, en tanto que un $70,00 \%$ de los profesores presentan una calidad regular, lo cual implica que la exhaustividad no tiene mayor prioridad en los profesores de la universidad.

Los aspectos que resultaron con mayores debilidades corresponden a la sinergia exhaustividad. A tal efecto, se analizan los aspectos predominantes de dicha sinergia, en razón de los resultados generados por un $70 \%$ de los profesores, quienes respondieron que rara vez profundizan los objetivos planteados en los contenidos de su unidad curricular, así como también, rara vez verifican las técnicas de aprendizajes con base a los objetivos formulados en la unidad curricular y facilitan material didáctico que permita estudiar a fondo los temas de la unidad curricular.

En este mismo sentido, un $65 \%$ de los profesores respondió que rara vez utilizan recursos didácticos para fortalecer los objetivos planteados y rara vez desarrollan técnicas de enseñanza que permitan comprender en su amplitud los temas de la unidad curricular.

Los resultados reflejan que la exhaustividad no es un factor determinante en el proceso académico de la universidad.

\section{Análisis de la sinergia innovación}

El cuadro 4 da evidencia que la sinergia innovación tiene un máximo de 48,3; un mínimo de 25,0 y una mediana de 41,667 puntos, por lo que se ubica, según el baremo de interpretación, en un rango de mediana calidad académica. La distribución es simétrica, por lo tanto, los grupos son homogéneos.

La distribución porcentual de la categoría innovación muestra en el cuadro 7 que un $31,4 \%$ de los docentes se encuentran en calidad deficiente y un $68,6 \%$ en calidad regular. De tal manera que los resultados no son favorables, pues ponen en evidencia la poca innovación de parte de los profesores que ejercen su función en la casa de estudio. Además, existe un porcentaje significativo $(31,4 \%)$ de profesionales que no son ávidos a producir, son docentes que no prestan un beneficio hacia la mejora de la comunidad educativa académica universitaria. 
Cuadro 7. Categoría de Innovación

\begin{tabular}{llcccc}
\hline & Frecuencia & Porcentaje & $\begin{array}{c}\text { Porcentaje } \\
\text { válido }\end{array}$ & $\begin{array}{c}\text { Porcentaje } \\
\text { acumulado }\end{array}$ \\
\hline Válidos & Calidad defi- & 22 & 31,4 & 31,4 & 31,4 \\
& ciente & & & & \\
& Calidad regular & 48 & 68,6 & 68,6 & 100,0 \\
& Total & 70 & 100,0 & 100,0 & \\
\hline
\end{tabular}

Los resultados correspondientes a la sinergia innovación que presentaron mayor debilidad estuvo conformada por un $65 \%$ de los profesores, quienes respondieron que algunas veces incluían técnicas de evaluación que contribuían a resultados novedosos dentro de la academia, de tal manera, se evidencia la monotonía del hacer académico y este, repercute en el aprendizaje de los estudiantes y en su capacidad de generar aportes innovadores.

Asimismo, un 68\% de los profesores respondió que rara vez facilitan técnicas de aprendizajes que fomentan la creatividad en la academia, así como también, rara vez incluye en la planificación de los contenidos las tecnologías de la información y la comunicación (Tic). Seguidamente, los profesores en un $63 \%$ manifestaron que rara vez incorporan en la planificación contenidos innovadores, ni trabajan de forma innovadora. Los resultados no favorecen la calidad académica de la institución universitaria, hoy día la innovación es un factor importante en las universidades.

\section{Análisis de la sinergia efectividad}

Con relación a la sinergia efectividad, en el cuadro 4 se observa que la misma muestra una mediana de 41,25 puntos de un máximo de cien puntos. Según el baremo de interpretación, se encuentra en un rango calidad académica mediana. La distribución es bastante simétrica, por lo tanto, los grupos tienen características similares, sin embargo, el grupo que está por debajo de la mediana presenta puntajes más dispersos que el que está por encima de la mediana.

Cuadro 8. Categoría efectividad

\begin{tabular}{|c|c|c|c|c|c|}
\hline & & Frecuencia & Porcentaje & $\begin{array}{c}\text { Porcentaje } \\
\text { válido }\end{array}$ & $\begin{array}{l}\text { Porcentaje } \\
\text { acumulado }\end{array}$ \\
\hline \multirow[t]{3}{*}{ Válidos } & $\begin{array}{l}\text { Calidad deficien- } \\
\text { te }\end{array}$ & 28 & 40,0 & 40,0 & 40,0 \\
\hline & Calidad regular & 42 & 60,0 & 60,0 & 100,0 \\
\hline & Total & 70 & 100,0 & 100,0 & \\
\hline
\end{tabular}

Con referencia a la distribución de la población en la categoría de la sinergia efectividad, los resultados señalan que un $40 \%$ de los profesores se ubican en la categoría de deficiente, lo que indica que hay un porcentaje importante de docentes que no son efectivos en su labor académica y un $60 \%$ de los profesores que realizan su trabajo con una calidad regular en correspondencia con la efectividad.

Los aspectos que demostraron más problemas en la calidad académica de los docentes estudiados se determinaron a través de las respuestas dadas por un $82 \%$ de los profesores quienes respondieron que rara vez planifican las actividades académicas tomando en cuenta el desarrollo de las competencias cog 
itivas, de la misma manera, respondieron que rara vez el trabajo del docente se orienta hacia el cumplimiento de las competencias procedimentales del PNF, y rara vez generan técnicas de aprendizaje efectivas como fundamento al desarrollo académico del programa nacional de formación.

Asimismo, un $83 \%$ de los profesores respondieron que rara vez contribuyen a facilitar contenidos que conduzcan al desarrollo general del programa nacional de formación. El $87 \%$ de los profesores respondieron que rara vez evalúan los recursos didácticos que estén orientados al desarrollo de las diferentes capacidades cognitivas para dar respuesta a las necesidades del programa nacional de forma- ción. Los resultados desfavorecen la mejora académica institucional universitaria.

\section{Análisis de la sinergia idoneidad}

De acuerdo al cuadro 4, la sinergia idoneidad tuvo un puntaje mínimo de 31,3; un máximo 52,5 y la mediana fueron de 43,750 puntos de 100, lo cual indica que medianamente los docentes realizan sus labores con rigurosidad exigidos de acuerdo al programa de formación. Los grupos estudiados según la distribución de los datos son homogéneos, es decir presentan características similares con respecto a los aspectos que resaltan en la sinergia.

Cuadro 9. Categoría idoneidad

\begin{tabular}{llcccc}
\hline & Frecuencia & Porcentaje & $\begin{array}{c}\text { Porcentaje } \\
\text { válido }\end{array}$ & $\begin{array}{c}\text { Porcentaje } \\
\text { acumulado }\end{array}$ \\
\hline Válidos & $\begin{array}{l}\text { Calidad de- } \\
\text { ficiente }\end{array}$ & 14 & 20,0 & 20,0 & 20,0 \\
& $\begin{array}{l}\text { Calidad re- } \\
\text { gular }\end{array}$ & 56 & 80,0 & 80,0 & 100,0 \\
& & & & \\
\hline
\end{tabular}

Los resultados del cuadro 9 demuestran que un $20,00 \%$ de los profesores se encuentra en calidad académica deficiente en cuanto a la sinergia idoneidad y un $80 \%$ de los profesores en calidad académica regular para la misma sinergia, esto indica que la labor académica universitaria no se realiza desde la idoneidad para acrecentar la calidad académica institucional.

Los resultados predominantes de la sinergia idoneidad se refieren a que un $70 \%$ de los profesores rara vez son claros y precisos, así como también, rara vez evalúan con claridad y precisión las técnicas de aprendizajes del programa nacional de formación para que su aplicación sea la más óptima. Un 73\% de los profesores dijeron que el desempeño docente rara vez es actualizado, como también rara vez, incluyen en su planificación contenidos actualizados de la unidad curricular.

Seguidamente, un $75 \%$ de los profesores expresaron que rara vez estudian los recursos didácticos para verificar si corresponden a las exigencias del programa nacional de formación y rara vez, evalúan los temas académicos para mantener actualizado el conocimiento dentro del programa nacional de formación.

Los resultados arrojan que la sinergia idoneidad es un componente poco relevante en el hacer académico de la universidad. Los docentes no tienen la disposición de trabajar con eficacia, eficiencia, lo que es evidencia de una mediana calidad académica. En tal sentido, prevalece lo negativo, pues la mayoría de los docentes no se enfocan en un trabajo competente que tenga como objetivo princi- 
pal la calidad académica universitaria. Por lo tanto, la idoneidad no se manifiesta en el desempeño académico del docente.

\section{Discusión de los resultados}

Los resultados obtenidos del evento calidad académica ubican a los docentes de la UPTT Manuela Sáenz en una categoría de mediana calidad académica, en razón de que alcanzó una mediana de 41,920 en una escala de 0 a 100 puntos. Esto refleja la existencia de un déficit en la calidad académica de los docentes que imparten sus unidades curriculares en dicha casa de estudio. La labor del docente, en cuanto a la calidad académica, se ve afectada porque no se trabaja con compromiso, dedicación, motivación, lo cual incide en el proceso de aprendizaje en los estudiantes. Además, una mediana calidad refleja que aspectos medulares de los procesos académicos se realizan sin considerar a profundidad la pertinencia, la exhaustividad, la innovación, la efectividad y la idoneidad.

Los resultados muestran disparidad con los planteamientos de Cano (1998), quien señala que hablar de la calidad de la educación no solo hace referencia a los procesos, sino también, al cumplimiento de cada uno de ellos, a la actividad pedagógica, asimismo, al compromiso de los estudiantes y a todos los actores, porque para lograr la calidad educativa es necesaria la intervención de todos sus actores. El profesor universitario y todo aquel que forme parte de la construcción del conocimiento, está en la obligación de ejercer su función con pertinencia, exhaustividad, innovación, efectividad e idoneidad, criterios que corresponden a la calidad académica.

En este sentido, si el docente se forma, se instruye constantemente, es un hecho que los estudiantes van a adquirir conocimientos novedosos que conducen a la productividad dentro y fuera de los espacios universitarios; el estudiante se direcciona hacía nuevos en- foques, hacia metodologías para abordar una investigación, de tal manera que la universidad se convierte en una gama de productos generado no solo por el docente, sino también por los estudiantes.

En este mismo orden de ideas, la calidad académica requiere de una labor responsable por parte de los dadores de conocimientos, la educación impartida debe estar acompañada de potenciales que permitan fortalecer la productividad del propio espacio universitario así como trascender los muros intrauniversitarios con el objeto de crecer como academia y generar cambios que se orienten hacia una transformación universitaria con calidad.

De igual manera, las autoridades de la UPTT Manuela Sáenz deben promover el progreso y desarrollo de la institución, transformar la academia, articular con sus mismos compañeros sobre nuevas técnicas pedagógicas, procedimientos, recursos didácticos que permitan generar consonancia entre el diseño curricular, las unidades curriculares, los avances científicos y tecnológicos, para generar solución dentro del ámbito académico y fuera de él.

Otro aspecto importante que promueve la calidad académica, es la investigación, siendo ella la columna vertebral de todas las universidades, en tal sentido, todos los profesores que están en el ámbito universitario tienen el deber de estar involucrado con la actividad investigativa, y es que, investigar conlleva a obtener conocimientos nuevos, a relacionarse con otros investigadores de diversas instituciones a nivel nacional e internacional, a publicar, a participar en congresos, simposios y otros eventos académicos, lo que trae un importante impacto en el realce profesional y el reconocimiento de la institución universitaria.

Con relación a los resultados de las sinergias se observó que todas se ubicaron en 
una categoría de mediana calidad académica, con valores de medianas que oscilaron ente 41,250 puntos y un máximo de 43,750 puntos de un máximo de 100 puntos; sin variabilidad significativa entre los valores en cada una de ellas.

Al estudiar el comportamiento de la sinergia pertinencia, puede observarse que la mediana fue de 42,500 puntos de un máximo de 100, ubicándose en el baremo de interpretación en una categoría de mediana calidad, lo cual evidencia desviaciones en el proceso académico de la UPTT Manuela Sáenz. En tal sentido, los resultados de la investigación en cuanto a la presente sinergia reflejan que los profesores de la universidad no ejercen una labor académica con pertinencia, la mayoría de los profesores no tienen el compromiso de ir hacia la mejora de la calidad, elemento fundamental dentro del trabajo académico, pues los conocimientos del profesor deben estar en correspondencia con las exigencias del programa de formación donde imparte.

Estos resultados contravienen la teoría de Tünnerman (2009), quien afirma que la pertinencia y la calidad deben trabajar integralmente, ya que la pertinencia da respuesta a un trabajo de calidad. Así, la UPTT Manuela Sáenz está obligada a replantearse, creativamente, sus objetivos, misión y funciones como institución de educación superior, para que este a la altura de las circunstancias actuales y del nuevo milenio. De igual manera, los docentes están en el deber de actualizar sus conocimientos, desarrollar sus habilidades técnico-profesionales, interpersonales y de solución de problemas, así como su actitud profesional, sus valores, normas de conducta, a fin de encontrar nuevas formas para adaptar sus funciones universitarias a las exigencias de la sociedad y dar respuesta a la misma.

A través del estudio realizado se comprobó que la exhaustividad arrojó una mediana de 42,50 puntos de un máximo de 100 puntos, correspondiente a una categoría de mediana calidad. Este resultado demuestra que la exhaustividad no es un factor relevante para los docentes de la universidad, es decir, el personal académico de dicha universidad no trabaja de forma profunda y objetiva en el desarrollo de su actividad académica, tal como el logro de las metas de la unidad curricular o las unidades curriculares que imparte o el abordaje extenso de los temas que conforman el programa nacional de formación donde pertenecen, lo cual conlleva a la desmejora de la academia.

Como puede notarse, existen discrepancias con relación a los datos de la sinergia exhaustividad y los postulados teóricos expresados por Vicuña y Hurtado (2014, p. 24) cuando plantea que "algo es exhaustivo cuando está completa, porque están incluidos todos los elementos que le son propias". En el caso planteado, el personal docentes de la institución referida al desarrollar el trabajo académico de manera objetiva y profunda adquiriría nuevas estrategias para su autoaprendizaje, mejoraría su creatividad y aplicaría mejores técnicas al momento de impartir el conocimiento a los estudiantes, razón de ser de la institución.

En referencia a la sinergia innovación presente en los docentes la UPTT Manuela Sáenz, la mediana fue de 41,667 puntos de un máximo de 100, por lo que se ubica según el baremo de interpretación en un rango de mediana calidad académica, lo que implica que los docentes de dicha institución rara vez incorporan aspectos innovadores en su labor académico ni trabajan de forma innovadora, siendo una debilidad para los docentes y por ende para la institución.

Estos resultados discrepan con los postulados teóricos de Oppenheimer (2014), quien considera que es de suma importancia crear una cultura de la innovación y fomentar la educación para la innovación en las institu- 
ciones universitarias. Obviamente, la cultura y la educación innovadora, se construyen desde que los dadores de conocimientos incluyen en su planificación académica contenidos actualizados, incorporan las tecnologías de la información y la comunicación como recursos en el apoyo del desempeño académico y como herramienta para estimular la creatividad.

Al estudiar la sinergia efectividad se halló una mediana de 41,250 puntos de un máximo de 100 puntos y se ubicó en correspondencia con el baremo de interpretación en el rango mediana calidad académica. Según estos resultados se infiere que los docentes realizan el quehacer académico con características de baja efectividad, así, rara vez orientan su trabajo hacia el cumplimiento de las competencias procedimentales del programa nacional de formación o con muy poca frecuencia generan técnicas de aprendizaje efectivas como fundamento al desarrollo académico.

Puede notarse que estos resultados son contrarios a la postura de Hunt (2009), quien considera que la efectividad en el docente debe partir del desarrollo adecuado en cuanto a sus competencias cognitivas, procedimentales y actitudinales, tomando en cuenta que cada competencia está dirigida a promover el trabajo idóneo y oportuno en la academia. Así, los logros y resultados académicos alcanzados permiten la incorporación de nuevos contenidos educativos para la práctica calificada, con resultados positivos en la labor académica de la institución universitaria.

En referencia a la sinergia idoneidad, esta presentó una mediana de 43,750 puntos de un máximo de 100 puntos, lo que le ubicó en correspondencia con el baremo de interpretación en el rango mediana calidad académica. Una mediana calidad académica denota que la institución universitaria presenta debilidades en cuanto a la idoneidad, tomando en consideración que la presente sinergia está referida al trabajo del docente realizado con los rigores exigidos por el programa de formación que se debe llevar en el ámbito académico.

Los resultados proyectados a través del estudio evidencian que el personal docente de la institución no se siente motivado, no aporta conocimientos nuevos, no conoce la filosofía del programa de formación al que pertenece y no se integra al quehacer universitario, es un profesional poco productivo que no está en la capacidad de formar los estudiantes críticos, responsables y comprometido con las necesidades de la sociedad contemporánea.

Estos resultados están en disparidad con la posición de expertos como Ramos, Fagúndez y Castells (2008) quienes dicen que, la idoneidad tiene criterios sistémicos de pertinencia, cuyo principal indicador empírico puede ser la adaptación entre los significados personales y los significados institucionales. De acuerdo a lo expuesto por el autor y los resultados proyectados a través del estudio, se evidencia que los profesores no están comprometidos con su labor pedagógica, esto crea un impacto negativo en la calidad académica universitaria.

\section{CONCLUSIONES}

La calidad académica de los docentes de la UPTT Manuela Sáenz los resultados la ubicaron en correspondencia con el baremo de interpretación en el rango mediana calidad académica, lo cual indica que la labor del docente, en cuanto a la calidad académica, se ve afectada porque no se trabaja con compromiso, dedicación, motivación lo cual incide en el proceso de aprendizaje de los estudiantes. Además, una mediana calidad refleja que aspectos medulares de los procesos académicos se realizan sin considerar a profundidad la pertinencia, la exhaustividad, la innovación, la efectividad y la idoneidad.

De igual manera, los valores de la sinergia pertinencia ubicaron a la institución en 
una mediana calidad académica, lo que denota poca pertinencia por parte de los docentes que desarrollan su quehacer académico en esta universidad, la mayoría de los docentes no tienen el compromiso de ir hacia la mejora de la calidad, elemento fundamental dentro del trabajo académico, ya que los conocimientos del profesor deben estar en correspondencia con las exigencias del programa de formación donde imparte y, este a su vez, con las necesidades del entorno o sociedad, es decir, no hay una adecuación entre lo que necesita la sociedad y lo que se enseña.

En la sinergia exhaustividad, los resultados la ubicaron en una categoría de mediana calidad académica, lo cual demuestra una desviación en la labor del personal académico de dicha universidad, quien no trabaja de forma profunda y objetiva en el desarrollo de su actividad académica, aspectos tales como el logro de las metas de la unidad curricular o las unidades curriculares que imparte o el abordaje extenso de los temas que conforman el programa nacional de formación donde pertenecen, son abordados someramente por los docentes, lo cual conlleva a la desmejora de la academia.

Asimismo, al estudiar la sinergia innovación los resultados ubicaron a la institución en una categoría de mediana calidad académica; se detectó que los docentes muestran apatía a la incorporación de los avances científicos y tecnológicos, tales como utilización de las Tics, incorporación de nuevas pedagogías, rigidez en cuanto a la aceptación de nuevas estrategias de aprendizaje creativo, entre otros aspectos, siendo una debilidad para el personal docente y por ende para la institución.

De acuerdo a la sinergia efectividad, nuevamente arrojaron una mediana calidad académica en la UPTT Manuela Sáenz; a través de la presente investigación se determinó que el trabajo realizado por los docentes de la insti- tución no se logra en su totalidad, mostrándose gran disparidad entre lo que se enseña el docente y el grado de utilidad que tiene para el desarrollo del estudiante. Estas debilidades traen como consecuencia poca credibilidad de la labor académica desarrollada en la universidad, así como apatía en los estudiantes y la deserción estudiantil

Por ultimo a la sinergia idoneidad que presentan los docentes de la UPTT Manuela Sáenz en correspondencia con el baremo de interpretación en el rango mediana calidad académica, lo cual indica que la labor del docente, en cuanto a la calidad académica, no se cumplió en su totalidad con las exigencias de los programas de formación que imparte la casa de estudio, de esta manera, no se logra una calidad académica institucional.

\section{REFERENCIAS}

Águila, V. (2005). El concepto calidad en la educación universitaria: clave para el logro de la competitividad institucional. Cuba: Dirección de postgrado, Ministerio de Educación Superior.

Cano, E. (1998). Evaluación de la calidad educativa. La Muralla, S.A.

Constitución de la República Bolivariana de Venezuela. (1999). Gaceta oficial de la República Bolivariana de Venezuela, 5.908. (Extraordinaria), 19-01-2009.

Crosby, P. (1987). La calidad no cuesta. El arte de cerciorarse de la calidad: México. Continental, S.A.

De la Fuente, J. (2008). Sociedad del conocimiento y la sociedad. Revista educación superior y sociedad. Caracas, Venezuela: Instituto Internacional de la UNESCO para la Educación Superior en América Latina y el Caribe IESALC.

Hunt, B. (2009). Efectividad del desempeño docente. Una reseña de la literatura internacional y su relevancia para mejorar la educación en América Latina. Programa de Promoción de la Reforma Educativa en América Latina y el Caribe (PREAL). Santiago, Chile. 
Kant, I. (2008). Sobre pedagogía. Argentina: Universidad Nacional de Córdoba.

Ley de universidades (1970). Gaceta Oficial $\mathrm{N}^{\circ} 1429$.

Llorens, F. (2013). En pos de la educación activa. Madrid: Cátedra UNESCO de gestión y política universitaria. Universidad Politécnica de Madrid.

Montes, A. (2013). Políticas de calidad de la educación en Iberoamérica. Universidad de Córdoba.

Morales, V., Medina, E. y Álvarez N. (2003). La educación superior en Venezuela. Informe 2002 a IESALC-UNESCO.

Oppenheimer, A. (2014). ¡Crear o Morir! La esperanza de Latinoamérica y las cinco claves de la innovación. Nueva York: Debate.

Ramos, A., Fagúndez, T y Castells, M. (2009). Los criterios de idoneidad y propuesta de cambios institucionales en el ámbito universitario. Revista Investigación y Postgrado. Vol. 24, número 3.

Salas, R. (2000). La calidad en el desarrollo profesional. Avances y desafíos. [Página web en línea]. Consultado el 10 de noviembre de 2016 en: http://scielo.sld.cu/ scielo.php?script=sci_arttext\&pid=S086421412000000200003

Tünnerman, C. (2009). Nuevas perspectivas de la pertinencia y calidad de la educación superior. Organización de las Naciones Unidas para la Educación la Ciencia y la Cultura.

UNESCO. (2008). La educación superior en América Latina y el Caribe: Diez años después de la conferencia mundial de 1998. Colombia: Organización de la Naciones Unidas para la educación, la ciencia y la cultura.

Vásquez, A. (2013). Calidad y calidad educativa. Universidad Nacional Mayor de San Marcos. Perú.

Vicuña, O. y Hurtado, J. (2014). Matriz para el Análisis de Trabajos de Investigación (Maiti). Manual. Cómo evaluar la calidad metodológica desde la comprensión holística de la investigación. Caracas, Venezuela: Inédito, Sypal. 